\title{
Research on method of BIT design for monitoring and alarm System of marine diesel engines power plant
}

\author{
Guo $\mathrm{He}^{1, \mathrm{a}}$, Xuedong Wen ${ }^{2,3} \mathrm{~b}$ and Kun $\mathrm{Bi}^{3, \mathrm{c}}$ \\ ${ }^{1}$ Dep. of Management Science, Naval Univ. of Engineering, Wuhan 430033, China; \\ ${ }^{2}$ College of Power Engineering, Naval Univ. of Engineering, Wuhan 430033, China; \\ ${ }^{3}$ Naval Equipment Repair Supervision Office in Qingdao area, Qingdao 266000, China. \\ ahgwxdong@163.com, bhg953760@163.com, cyingshiye_wen@qq.com
}

Keywords: diesel engine power plant, monitoring and alarm system, DFT, BIT.

\begin{abstract}
In view of the deficiency of design for testability (DFT) of the monitoring and alarm system of marine diesel engine power plant (MASMDEPP), this paper offers an overall built-in test (BIT) scheme which can improve the level of DFT for the system. Based on analysis of nine kinds of line replaceable units (LRU), the paper gives the detailed design of BIT hardware circuit and develops a model machine for a MASMDEPP. Under the laboratory conditions as well as real ship tests, the performance indexes of the prototype can meet all of the design requirements. This study is of great significance for improving the fault diagnosis ability of a certain type of MCSMDEPP, and lays a foundation for the application of DFT technology in the monitoring system of marine platform.
\end{abstract}

\section{Introduction}

With the development of microelectronic control technology, communication bus technology and manufacturing technology, the function of MCSMDEPP is becoming more and more powerful, and the structure is becoming more and more complex. Naturally, the attendant problem of testing and diagnosis is also becoming more and more prominent. At present, the test and diagnosis design of chip level or module level can only realize fault diagnosis for a single LRU of system, which cannot test all of the LRUs and theirs mutual influence in the system. Moreover, it is unrealistic for on-site testing and maintenance that to equip with a large number of technical personnel and External Test Equipment (ETE) in marine engine room.

Aiming at the above problems, DFT technology considers in parallel about the problems of test and diagnosis in the stage of equipment demonstration, design and development, whose purpose is to reduce life cycle cost, improve rate of combat readiness and mission success for the equipment. It can equip the equipment with an innate ability of fault detection and isolation at a lower cost, which can achieve efficient and accurate determination of the state of the system (work, cannot work or performance degradation) and isolate its internal faults [1].

At present, the application of DFT technology for the domestic manufacturers of MCSMDEPP has not been carried out intensively. Moreover, the concerning research work for many devices is still blank. According to the requirement of work item 305 in literature [2], For the specific product system, The DFT scheme needs to consider the general testability criteria and the system itself characteristics, and then transforms the requirements of testability, the constraints of use and maintenance into specific test projects, such as BIT, ETE and manual test equipment (MTE), etc. In this paper, a MCSMDEPP is taken as the object, and the overall scheme of DFT of the system is put forward, and the detailed BIT design method of the system is studied.

\section{Overall scheme of DFT for a MCSMDEPP}

According to the system structure of a MCSMDEPP, this paper presents the of a distributed-centralized overall scheme of DFT based on field bus technology by using BIT, ETE and 
MTE, as shown in Fig. 1. During the testing process, the function of BIT is to detect system fault and isolate it to a certain LRU, which may not be detected by ETE or MTE. Thus, BIT, ETE and MTE are closely related to each other. Therefore, the functional complementarity and interface compatibility among three of them must be carefully taken into account through the designing process.

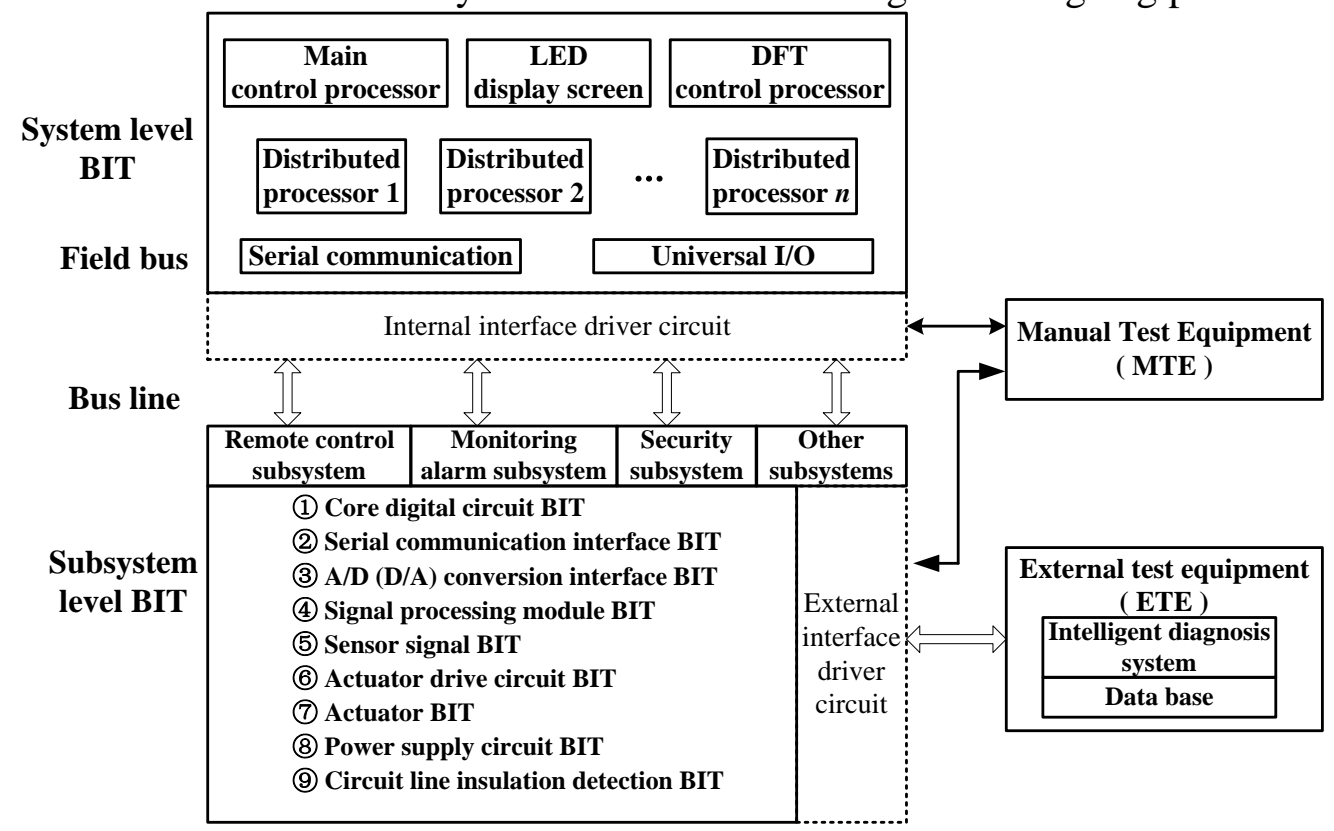

Fig.1 Hardware structure diagram of DFT improved scheme for MCSMDEPP

This scheme takes advantage of CAN bus in the field of distributed control and interoperability, and each BIT node of subsystem level is connected to the measurement and control network using bus protocol. It is beneficial to the miniaturization and networking of the test system, and the failure of any BIT node affects only the node itself without endangering the whole system.

\section{Hardware failure mode analysis in LRU level and its BIT design}

On the basis of studying the structure and principle of a MCSMDEPP, This paper divides the system into 9 kinds of LRU type which are as follows: power supply circuit, circuit line, sensor, signal processing module, A/D (D/A) conversion interface, core digital circuit, serial communication interface, actuator drive circuit, and actuator. In addition, failure modes of each LRU are listed respectively as well as their design scheme of BIT.

\subsection{Failure mode analysis and BIT design for core digital circuit}

The core digital circuit of MCSMDEPP control node is MCU and its external memory (RAM/ROM). There are many kinds of faults in MCU, such as register fault, instruction decoder failure, data processing error, and so on [3]. Although the fault reason is various, the faults are represented as not executing the program, not outputting the instruction, or incorrect data. The BIT method for VLSI usually adopts BIT of microprocessor and boundary scanning [1]. Without modifying the internal design of the circuit under test, MCU fault can be diagnosed by detecting specific pins of clock/reset circuit, Watchdog timer and the command output port.

RAM/ROM fault mainly includes read / write logic fault, memory sequence fault, decoding error, interference fault and so on, of which the common features are read and write errors. The above fault modes can be verified by running the RAM/ROM unit summation detection, cyclic redundancy check and parity check [1].

\subsection{Failure mode analysis and BIT design for serial communication interface}

The signal processing module of MCSMDEPP can upload or share parameters of the diesel engine to other network nodes through serial interfaces of CAN network. The failure modes of serial communication interface circuit are generally reflected with unchanged signal value and open circuit, etc. [4]. They can be tested by sending or receiving auto feed detection on the hardware or setting the check bit on the software. By using fault self-test program of FCS, the communication state of each 
node can be detected quickly, and fault node can be automatically suspended to isolate the fault. The technical characteristic of FCS provides an advantage for BIT design in MCSMDEPP.

\subsection{Failure mode analysis and BIT design for A/D (D/A) conversion circuit}

Fault test for A/D (D/A) conversion circuit can be completed by the joint wrap comparator method [5], as shown in Fig. 2. When testing A/D conversion circuit, it is necessary to read the preset voltage signal and convert it, then compare the result with the standard value. When D/A channel is tested, the output of the D/A is read into the $\mathrm{A} / \mathrm{D}$ channel through the multiplex switch, and the conversion result is compared with the preset value.

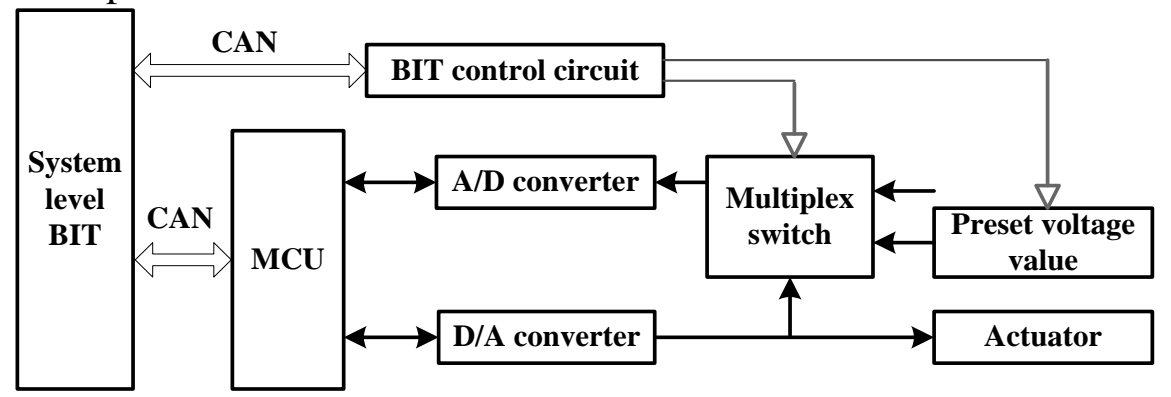

Fig. 2 Schematic of loopback test for A/D-D/A converting circuit

\subsection{Failure mode analysis and BIT design for sensor signal processing module}

There are five types of sensor signal processing modules in MCSMDEPP. Because the different these types of circuits are different, the failure modes will also have different characteristics. However, the general failure of these modules can be summed up as drift of measurement value; chip failure; clock / reset circuit failure; short circuit and open circuit; etc. Consider to the above fault types, this paper uses method of wrapping contrast detection to verify the effectiveness and accuracy of output signal. In this method, so as to realize the function detection of the 5 kinds of modules, a standard excitation signal is firstly inputted, then the output signal is compared with the standard output signal. The test principle is shown in Fig. 3.

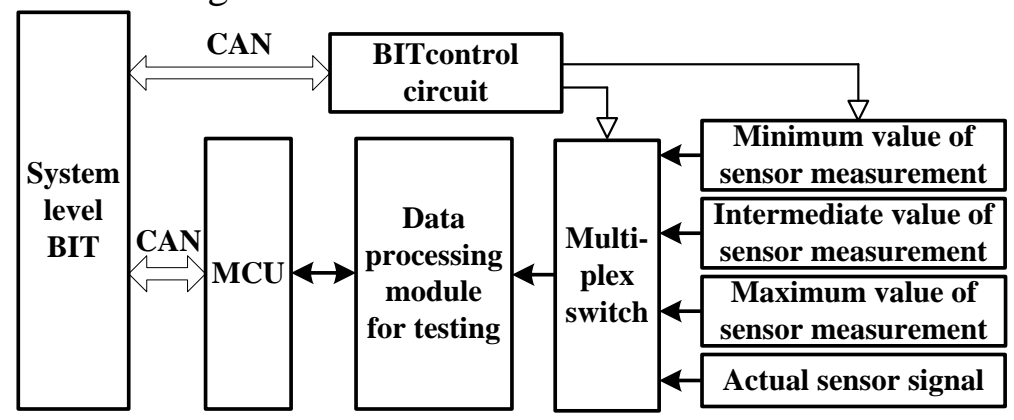

Fig.3 Schematic of loopback test for signal processing module of sensors

\subsection{Failure mode analysis and BIT design for sensors}

The types of MCSMDEPP sensors are divided into pressure sensor, pressure difference sensor, temperature sensor, temperature difference sensor, pulse speed sensor, displacement sensor, switch sensor, liquid level sensor, etc. Although there are many kinds of sensors, the fault types can be divided into two categories: hard fault and soft fault [6]. Hard fault refers to the sensor output signal over its own or the physical limits of the object, of which the form is signal mutation or no change. The soft fault is that the sensor signal does not exceed the physical limit of the measured object, which mainly shows the measurement of drift, uncertainty, etc.

For hard faults, methods, such as signal range threshold detection, signal change rate detection and signal comparator detection, can be used to confirm the faults. The signal range threshold detection is to detect whether the sensor is out of range by comparing the sensor output signal and the effective output threshold value, and then determine whether the sensor is faulty. The signal change rate detection is measured by comparing adjacent signals value collected by a sensor and calculating the rate of change, then determines whether the sensor beyond the measurement range or not. For soft fault, the redundant sensors can be set up at the important measuring points by comparing the measured values with a standard signal. 


\subsection{Failure mode analysis and BIT design for actuator drive circuit}

Combined with the current state parameters of the diesel engine, the remote / security controller of MCSMDEPP can output the control signal according to the given command, and drive the actuator to complete the specified action.

The paper use method of wrapping contrast to detect failures of actuator drive circuit. As shown in figure 4, the BIT control circuit receives the output signal of the actuator drive circuit, and compares it with the expected value of the standard output signal to determine whether the drive circuit is faulty.

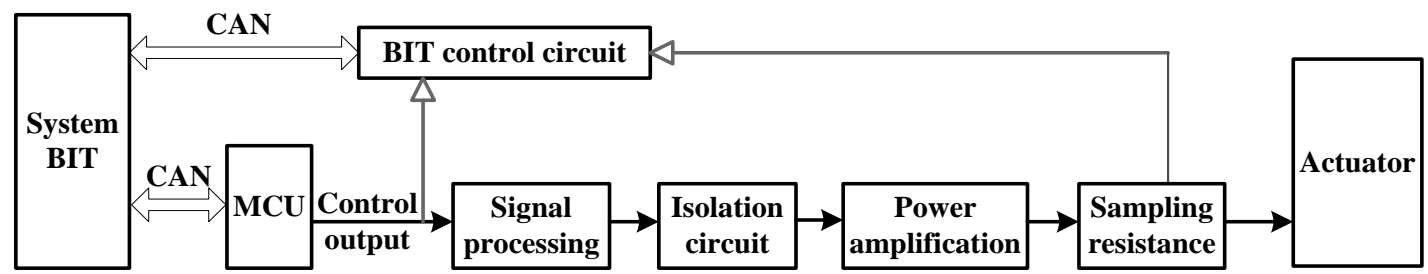

Fig.4 Schematic of loopback test for actuator driver circuit

\subsection{Failure mode analysis and BIT design for Actuator winding coil}

According to the signal type, actuator drive circuit of MCSMDEPP can be divided into two classes, switch and analog. Both of them respectively use solenoid valve, electrical (liquid) servo valve and displacement actuator, forming double mechanical, angular or linear displacement. The core electric components of the above two electromagnetic drive circuits are electromagnetic winding coils [7]. Due to the high temperature, high humidity and strong vibration of the ship working environment, within the electromagnetic winding, short circuit, circuit breaker, insulation failure and other failures often happen, which results in the failure of the actuator.

Considering the electromagnetic winding access feedback signal line which will reduce the reliability of the circuit, BIT design should not be used directly to change physical structure of electromagnetic coil. Instead, it uses the method of off-line voltage comparison to respectively detect the short circuit, open circuit and insulation of the electromagnetic winding, as shown in Figure 5. When BIT is working, the testing system connects the insulation path of the winding, and the inspection signal is input into the actuator circuit. The input signal strength (12V) is not sufficient to cause the actuator to produce an action, but can give the results of "open circuit, short circuit or insulation". In the process of detection, the short circuit fault and the open circuit fault of winding coil are detected, and then the insulation condition of winding coil is detected.

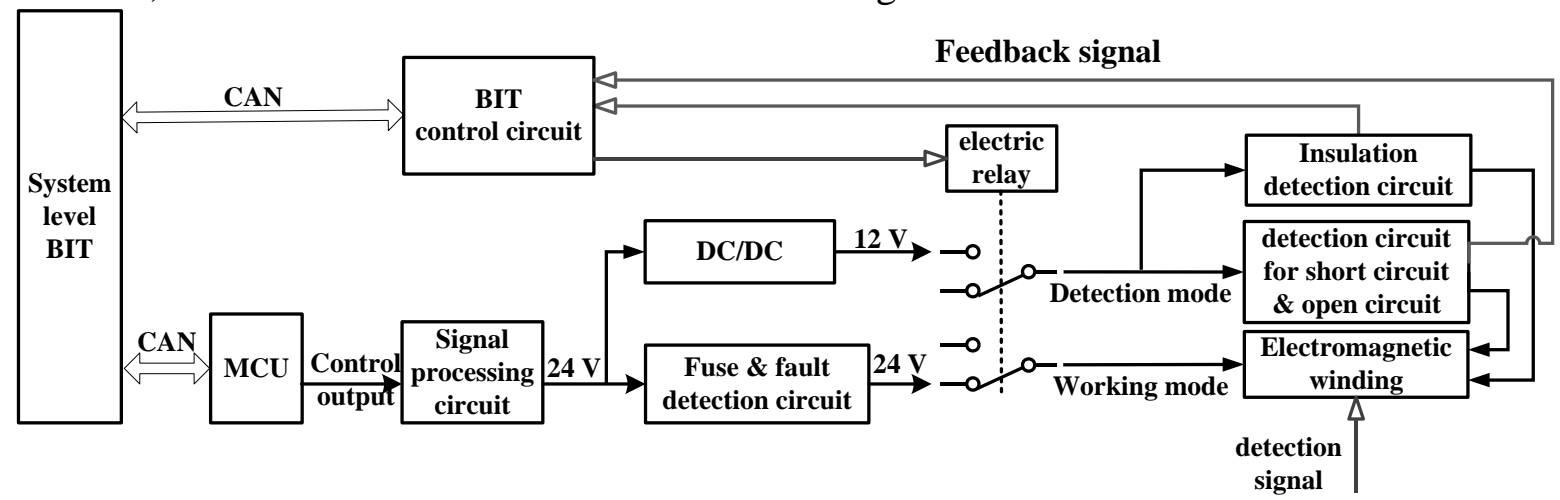

Fig.5 Schematic of voltage contrast test for electromagnetic winding of actuator

\subsection{Failure mode analysis and BIT design for power supply circuit}

In MCSMDEPP, the power of each subsystem is required from the AC power network of ship by $\mathrm{AC} / \mathrm{DC}$ conversion. And then on the basis of specific requirements of different equipment, secondary powers convert into working voltage through DC/DC voltage converter. Because the power system fault is the most common fault in the ship engine room equipment, the power supply circuit is usually designed with double redundancy.

Comparative voltage detection method based on redundancy technology is usually used in power supply circuit fault detection, as shown in Figure 6. For the AC input of the system level, the voltage of the main and standby power supply circuits is compared by using the marine power own redundancy 
design to judge whether the input voltage is normal. Aiming at the fault detection of DC low voltage power supply, it is necessary to compare it with the given reference voltage, so as to judge whether the input voltage of DC meets the requirement of power supply.

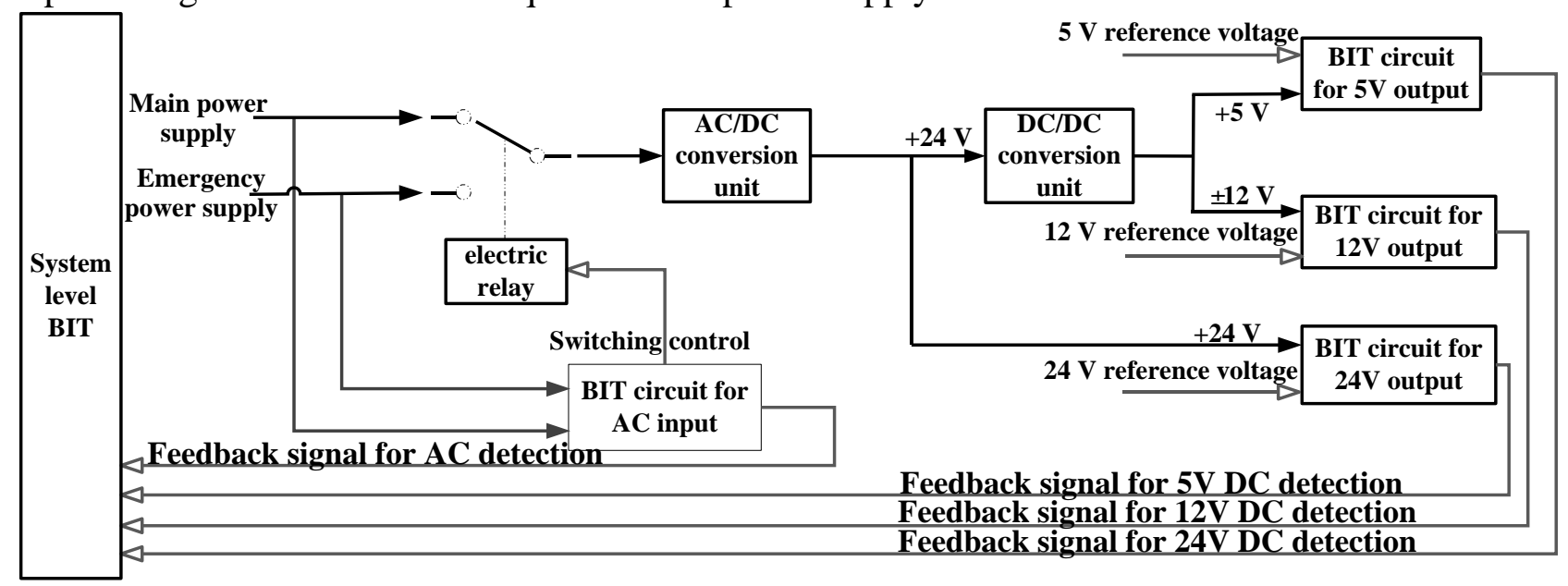

Fig.6 Schematic of BIT for system's power supply circuit

\subsection{Failure mode analysis and BIT design for circuit line insulation detection}

In addition to the application of the bus cable for digital signal transmission, within the low voltage DC power supply, sensor and signal processing module, the actuator and the driving circuit, the circuit transfer box and other positions, there are still a large number of dense and complex analog transmission lines in MCSMDEPP. Although the fault detection principle is relatively simple, it is one of the most difficult to diagnose faults.

In general, the good insulation performance of the electronic circuit is the premise to ensure the reliable operation of electronic equipment, and the decline of line insulation is one of the precursors of the short circuit fault. Under the condition of BIT insulation detection, the system automatically sends the inspection signal to the circuit to be tested, and the signal strength will not break the circuit performance. But it can be concluded that the circuit insulation resistance state.

Figure 7 is the basic principle of detecting the insulation resistance of a line in the DC low voltage power supply network. By setting the reference voltage Vs, after the start of the test, the signal generator sends out the detection signal, and the data processing circuit receives V0, then V0 and Vs are compared until the testing conclusion is obtained. After the detection of a certain path, through the multi-channel switching unit, it can be achieved on insulation detection of all lines (including positive and negative wiring).

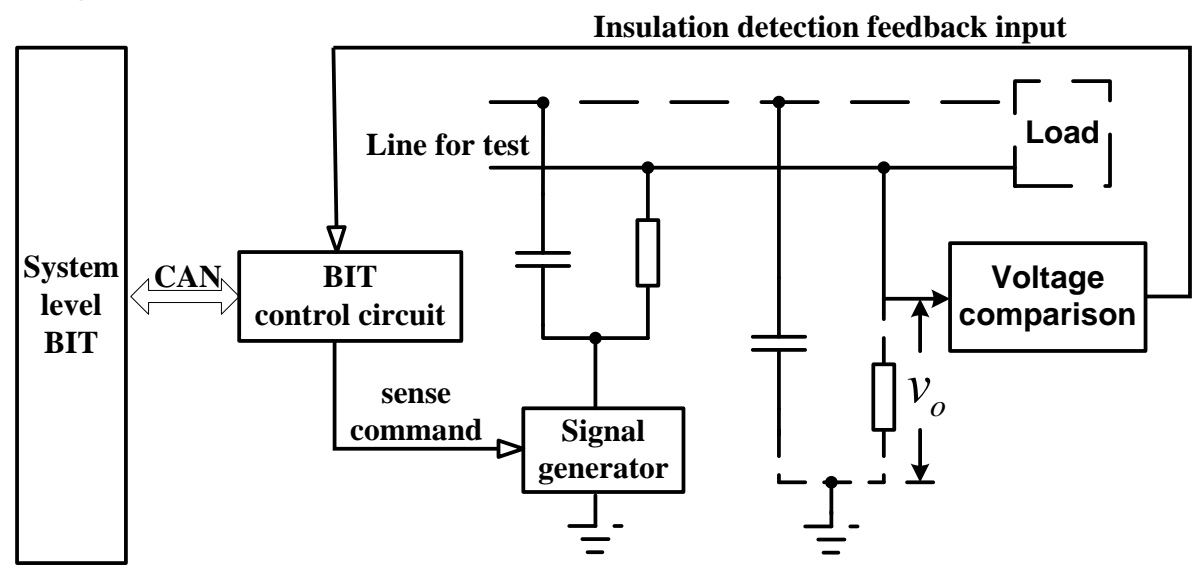

Fig.7 Schematic of insulation detection based on comparison of reference voltage

\section{Summary}

With the continuous development of engine room monitoring technology, DFT should be considered as an important part of the whole system design, which must be considered from the design 
stage. Especially in design and development phase of the MCSMDEPP system, the BIT technology as the most important technology in DFT should be strengthened. This paper focuses on the DFT improvement needs of MCSMDEPP, and presents a distributed-centralized BIT design scheme based on FCS technology. Moreover, taking a certain MCSMDEPP system as the research object, this paper describes in detail about the BIT design method of nine LRU level units which are composed of the system. Finally, the performance and function of the prototype are tested in the laboratory and real ship condition. The results show that the performance and the function of the prototype meets the design requirements, which can fully verify effectiveness and credibility of the BIT design.

\section{References}

[1]. J. Qiu, G.j. Liu, P. Yang, et al.Modeling and designing of testability technology for equipment, Science Press, 2012, p. 1-5.

[2]. GJB 2547A. General requirements of testability for equipment. National Defense Military Standard Press, 2012, p. 1-2.

[3]. Chunjiang Bian. The Design and Validation of BIT for Aero-engine Electronic Controller, Nanjing University of Aeronautics and Astronautics, China. 2005, p. 45-49.

[4]. Li Xuanjun. Research of BIT Technology for Aero-engine Digital Controller and Integrated System of Avionics. Nanjing University of Aeronautics and Astronautics, China. 2001, p. 112-118.

[5]. Jianjun Han. Design and Validation of a Dual-channel and Fault-tolerant EEC based on BIT. Nanjing University of Aeronautics and Astronautics, China. 2010, p. 76-78.

[6]. Walker R K. Built-in-test Instrumentation and 21 Rules of Thumb. IEEE Instrumentation and Measurement, 2002, p. 30-38.

[7]. Haisong Liu, Jiechang Wu, Guocai Yang, et al. Research on BIT Design of Engine Room Monitoring and Control System. Machinery and Electronics. Vol. 7 (2012), p.48-51. 